\title{
The RF performance of cavity made from defective niobium material determined by Eddy Current Scanning
}

\author{
G. $\mathrm{Wu}^{1}$, L. Cooley ${ }^{1}$, D. Sergatskov ${ }^{1}$, J. Ozelis ${ }^{1}$, A. Brinkmann ${ }^{2}$, W. Singer ${ }^{2}$, X. Singer ${ }^{2}$ and M. \\ Pekeler ${ }^{3},{ }^{1}$ Fermilab, ${ }^{2}$ DESY, ${ }^{3}$ RI Research Instruments $\mathrm{GmbH}$
}

\begin{abstract}
Eddy current scanning (ECS) has been used to screen niobium sheets to avoid defective material being used in costly cavity fabrication. The evaluation criterion of this quality control tool is not well understood. Past surface studies showed some features were shallow enough to be removed by chemical etching. The remaining features were identified to be small number of deeper inclusions, but mostly unidentifiable features (by chemical analysis). A real cavity made of defective niobium material has been tested. The cavity achieved high performance with comparable results to the cavities made from defect free cavities. Temperature mapping could help to define the control standard clearly.
\end{abstract}

Index Terms-Accelerators, Eddy current scanning, Superconducting accelerator cavities, Superconducting RF, Surface treatment.

\section{INTRODUCTION}

E DDY Current Scanning (ECS) has been used to screen niobium sheets to avoid potentially defective material from being used in costly cavity fabrication $[1,2]$. The evaluation criterion of this quality control tool is not well understood. Investigating a niobium cavity made of defective niobium material could help to clearly define the control standard, thus potentially reducing the overall material cost without increasing the risk of fabricating a defective cavity.

A recent batch of niobium sheets provided by a manufacturer showed a high percentage of sheets with noticeable features as detected by ECS equipment. These ECS features vary in number, size and shape. Past surface studies indicate some features were shallow enough to be removed by chemical etching. The remaining features were identified by chemical analysis and found to be a small number of deeper inclusions, with the balance being mostly unidentifiable features. Past cavity tests showed tantalum inclusions could cause cavity performance degradation [1]. But many features

Manuscript received 3 August 2010. This work was supported in part by the U.S. Department of Energy under contract number DE-AC02$07 \mathrm{CH} 11359$.

G. Wu, L. Cooley, D. Sergatskov and J. Ozelis are with the Fermilab, Batavia, IL 60510 USA (phone: 630-840-5409; e-mail: genfa@fnal.gov).

A. Brinkmann, W. Singer and X. Singer are with DESY, Hamburg, Germany.

M. Pekeler is with RI Research Instruments, GmbH, Bergisch Gladbach, Germany. identified by ECS were not identified as tantalum inclusions and no studies have been done to show the correlation between these other features and RF performance.

Two cavities were manufactured using sheets with potential defects as determined by ECS equipment. One cavity was tested yielding high accelerating gradient comparable to the cavities made from feature-free niobium sheets.

\section{NIOBIUM MATERIAL AND CAVITY}

Two niobium sheets were visually inspected and no visible defects were identified. They were scanned by ECS equipment on both sides. The ECS equipment used an RF frequency of $200 \mathrm{KHz}$ in one channel. Such frequency corresponds to a penetration depth of $0.35 \mathrm{~mm}$ for niobium material. Figure 1 and 2 showed the ECS images of two sides of one niobium sheet. The large dot feature on RF side of sheet FE234 is also visible on the non-RF side. This indicates the feature is large and could be detected by the eddy current scanner on both sides of the sheet.

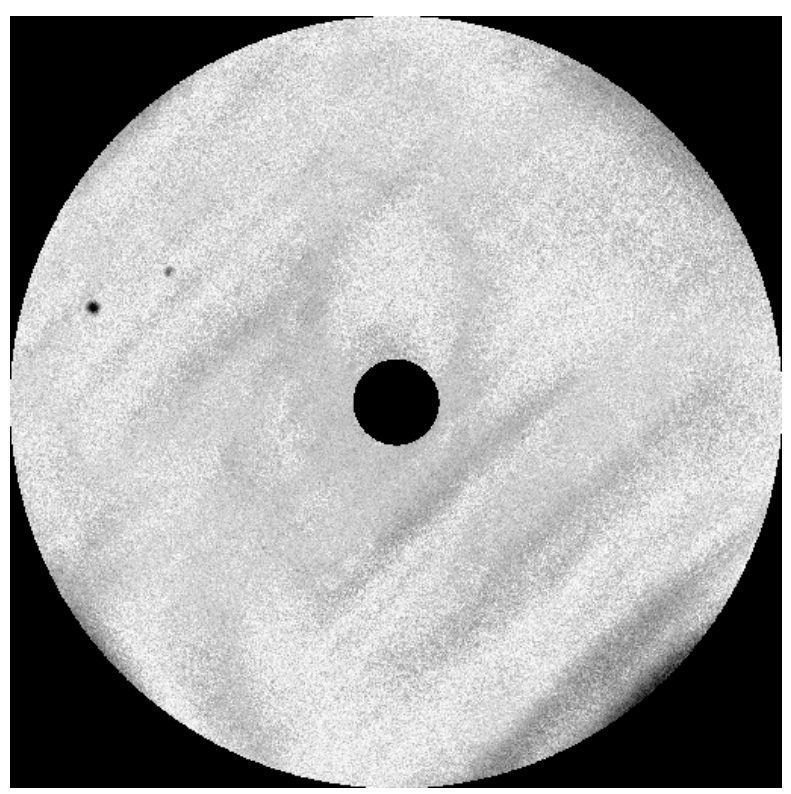

Figure 1: ECS image of RF side of niobium sheet FE234 in cavity TE1ACC005.

Although the eddy current scanner does not have information about the depth of the feature, by adjusting the 
scanner frequency one can obtain some information based on penetration depth of niobium material. Unfortunately this option is not ideal as the scanner electronics were optimized for a certain frequency range. The decreased image quality prevented us from resolving the exact feature depth. Nevertheless, the feature on the sheet can still be detected after changing the eddy current scanner operating frequency to 600 $\mathrm{KHz}$. From this we can conclude that, the feature must be located in the sheet at a depth of $200 \mu \mathrm{m}$. Standard cavity processing removes niobium material in the range of $150 \mu \mathrm{m}$ to $250 \mu \mathrm{m}$. The feature is located close to the outer edge of the sheet, where the magnetic field is the strongest in a TESLAshaped cavity. The feature is located in an area such that it is not in the electron beam weld path and heat affected zone. This niobium sheet FE234 is indeed an ideal sheet to be made into 1-cell cavity.

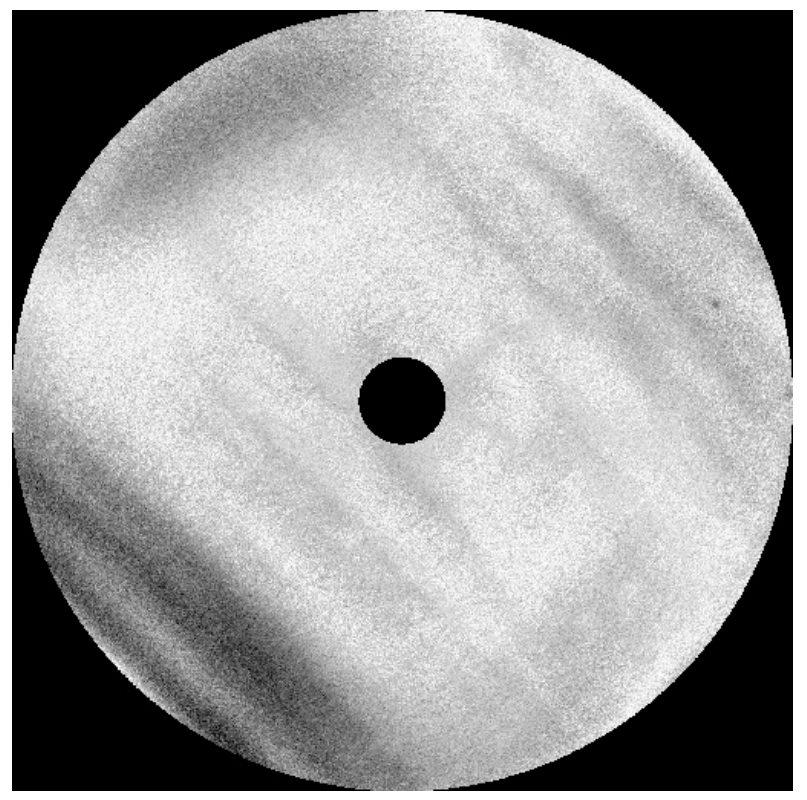

Figure 2: ECS image of non-RF side of niobium sheet FE234 in cavity TE1ACC005.

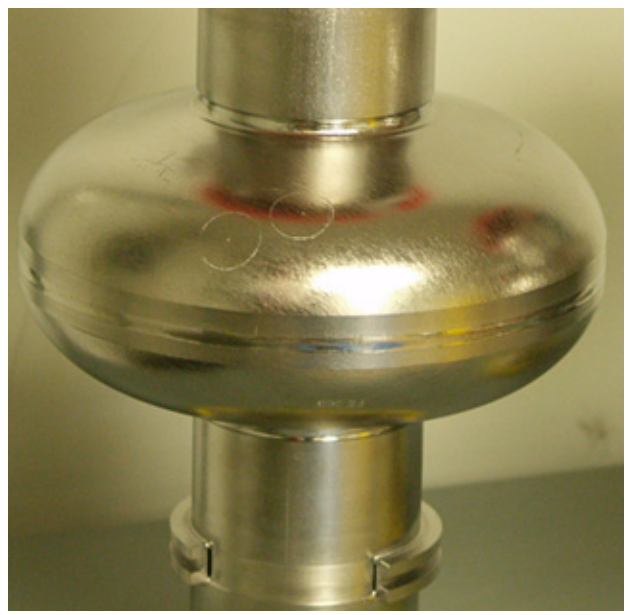

Figure 3: 1-cell cavity TE1ACC005 with the ECS feature marked in circles on the external surface.
Cavity TE1ACC005 was manufactured using standard niobium cavity procedures. Niobium sheet FE234 and another ECS feature free sheet were paired together to form the final 1-cell cavity. Figure 3 shows the cavity TE1ACC005 with the feature location marked on the external surface. Upon receipt from manufacturer, the internal weld and the ECS feature location were optically inspected using a high resolution camera [3]. No identifiable feature was found.

\section{CAVITY PROCESSING AND RF MEASUREMENT}

\section{A. Chemical processing}

During the cavity manufacturing, sheets were deep drawn and prepared for electron beam weld. About a $20 \mu \mathrm{m}$ thick layer of niobium material was removed through BCP processing before the first weld. Another $30 \mu \mathrm{m}$ of material was removed by BCP processing before the cavity was finally welded.

After a thorough optical inspection, the cavity was processed using standard electropolishing. A thickness of 40 $\mu \mathrm{m}$ of niobium was removed. The cavity was then tested in the standard fashion in a $2 \mathrm{~K}$ liquid helium bath.

After this test, another $40 \mu \mathrm{m}$ of niobium material was removed through standard EP process, followed by a $120{ }^{\circ} \mathrm{C}$ bake out and a second RF test. Test results are discussed in next section.

\section{B. Temperature mapping and cavity performance}

During the first cavity test, eight thermometers were mounted along the equator, evenly distributed. An additional four thermometers were attached to each of the ECS feature locations as shown in Figure 4.

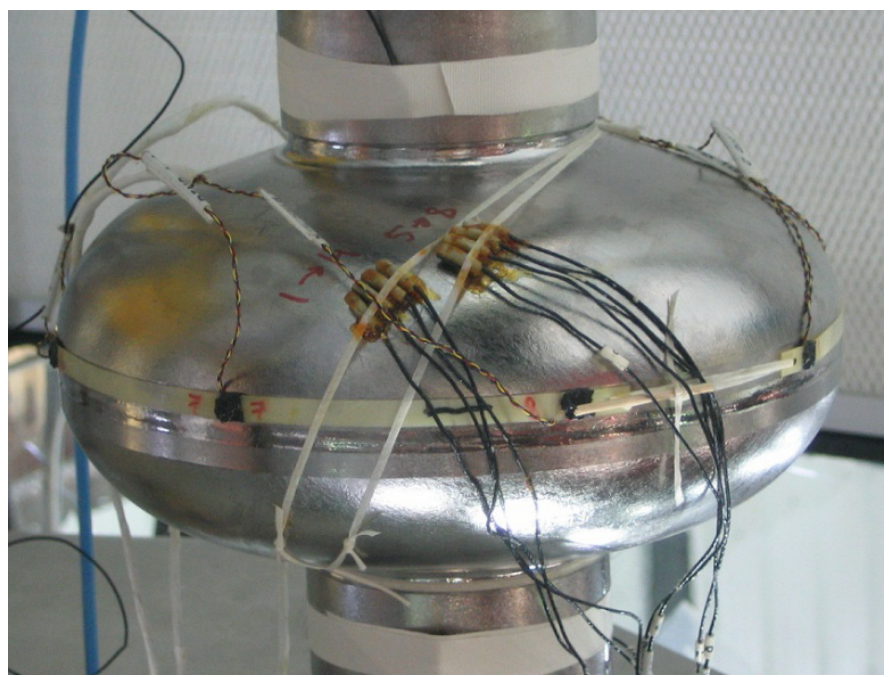

Figure 5: 1-cell cavity TE1ACC005 with 16 thermometers distributed on equator and two ECS feature locations.

During the first test, the cavity reached $38 \mathrm{MV} / \mathrm{m}$ (limited by a quench) with good quality factor of $1 \times 10^{10}$, as shown in Figure 6. No temperature rises were detected by any of the temperature sensors attached to the two ECS locations. One of the equator thermometers registered a high temperature rise at 
the quench field. The location was far away from the ECS locations.

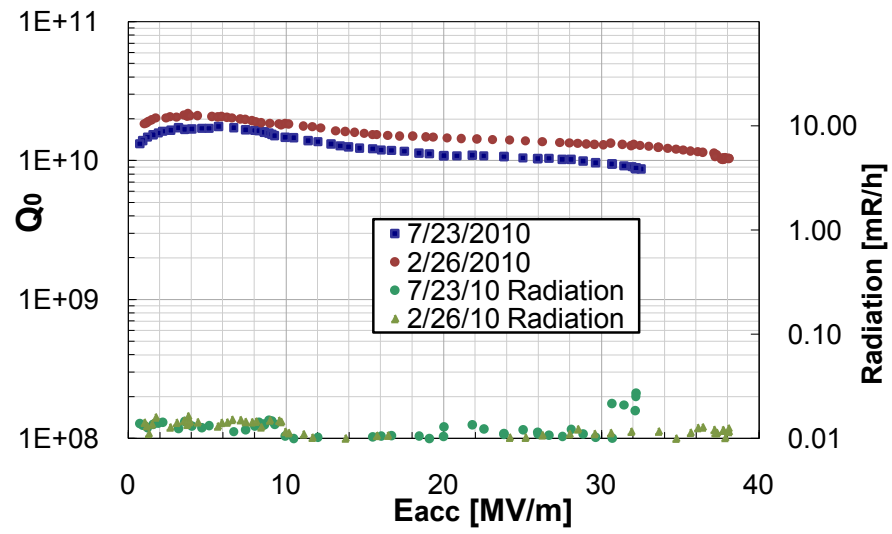

Figure 6: Q vs. Eacc measured for two RF tests.

During the second RF test, only 8 thermometers were attached to cavity equator. The cavity accelerating gradient reached $32.5 \mathrm{MV} / \mathrm{m}$. One of the equatorial thermometers detected a high temperature rise during quench. Again, that sensor location was about $13 \mathrm{~cm}$ away from the location of the features identified by the ECS. Although the cavity performance was lower than that of the first RF test, thermometer data conclusively showed that the ECS feature did not limit the cavity performance.

\section{CONCLUSION}

Cavity tests with thermometers attached to the identified ECS feature locations demonstrated that the impact of the ECS detected features on cavity performance is not completely understood. Since the depth of the ECS detected features is not well known, it cannot be assumed a priori that they are harmful. Since it is possible the feature can be deeply buried in the niobium material, further material removal might indeed uncover the potential defects of the niobium sheet which then may affect the RF surface during a subsequent test, leading to poorer cavity performance. The ECS should continue to be used as general quality assurance tool to safeguard the potential defective niobium material for cavity production.

\section{ACKNOWLEDGMENT}

The authors would like to thank the general cavity support from A. Rowe, D Bice, C. Baker, B. Stone, T. Reid and R. Murphy. We also thank K. Swanson for editorial help.

\section{REFERENCES}

[1] W. Singer, A. Brinkmann, D. Proch and X. Singer, Physica C: Superconductivity, Volume 386, 15 April 2003, Pages 379-384.

[2] C. Boffo et al, IEEE Transactions On Applied Superconductivity, VOL. 17, NO. 2, June 2007.

[3] K. Wanatabe, "Review of Optical Inspection Methods and Results," in Proc. of 14th Workshop on RF Superconductivity, Berlin, 2009, pp. 123128. 